I. Stamatellos $\cdot$ P. Stamatopoulos $\cdot$ D. Rousso

E. Asimakopoulos · C. Stamatopoulos · I. Bontis

\title{
Investigation of abnormal uterine bleeding in perimenopausal women by hysteroscopy and endometrial biopsy
}

Received: 16 August 2004/ Accepted: 28 November 2004/Published online: 18 February 2005

(C) Springer-Verlag Berlin / Heidelberg 2005

\begin{abstract}
The causes of abnormal uterine bleeding (AUB) during the perimenopausal period in women receiving hormone replacement therapy (HRT) were investigated, along with the causes of postmenopausal bleeding, the efficacy of hysteroscopy in the diagnosis of endometrial pathology was investigated. The patients studied were referred to our academic gynaecological clinic during the period 1999-2003. 145 cases that had undergone hysteroscopy and endometrial biopsy for AUB during the perimenopausal period over the last four years were reviewed retrospectively. The main group (76 patients) presented AUB while they were taking sequential or continuous HRT for menopausal symptoms and to prevent osteoporosis. The control group (69 patients) presented postmenopausal bleeding. There was an increased incidence of endometrial pathology in the group receiving HRT in comparison with the control group $(52.6 \%$ vs. $40.4 \%)$, but this difference was not significant. The main findings were functional endometrium $(38.1 \%)$ in the group receiving HRT and atrophic endometrium (40.6\%) in the control group, respectively. These differences were statistically significant between the two groups. Diagnostic hysteroscopy was performed successfully with no serious complications and was performed without anesthesia in over $30 \%$ of the patients in both groups. The incidence rates of the structural endometrial causes of AUB in perimenopausal period are similar in women taking
\end{abstract}

\footnotetext{
I. Stamatellos $(\varangle) \cdot$ P. Stamatopoulos $\cdot$ E. Asimakopoulos C. Stamatopoulos I. Bontis

First Department of Obstetrics and Gynaecology, Papageorgiou General Hospital, Aristotle University of Thessaloniki, Periferiaki Odos, Nea Eukarpia, P.C.54606 Thessaloniki, Greece

Tel.: + 2310693131

Fax: +2310991510

E-mail: stamio@mail.com

D. Rousso

Third Department of Obstetrics and Gynaecology, Hippokrateion General Hospital, Aristotle University of Thessaloniki, Thessaloniki, Greece
}

HRT and those presenting postmenopausal bleeding. Hysteroscopy is a safe and efficient method of investigating women with AUB during the perimenopausal period. In addition, the increasing use of outpatient hysteroscopy will simplify assessments of patients presenting with AUB during the perimenopausal period.

Keywords Hysteroscopy - Abnormal uterine bleeding Endometrial biopsy $\cdot$ Hormone replacement therapy

\section{Introduction}

The mean age of menopause in women is between 50 and 52 years (51.3 years) [1]. 2-8 years before menopause, most women present anovulatory cycles with long proliferative phases [2] and reduced numbers of follicles $[3,4]$. Abnormal uterine bleeding (AUB) during the perimenopausal period consists of one or a combination of disorders in frequency, predictability, duration, and rate of flow, and may be accompanied by related symptoms of fatigue and dysmenorrhea. AUB associated with the use of sequential or continuous HRT is the main reason for patient dissatisfaction and interruption of this therapy. The objective of this study was to investigate, by hysteroscopy and endometrial biopsy, the causes of AUB in perimenopausal patients on sequential or continuous HRT regimens by comparing them with the causes of postmenopausal bleeding observed in another group of patients. In addition, this study emphasizes the increasing use of outpatient hysteroscopy as an effective and acceptable method for treating patients with bleeding problems [5].

\section{Subjects and methods}

A total of 145 perimenopausal patients exhibiting AUB during the use of HRT or presenting postmenopausal bleeding were included in this study. The mean age of 
the patients was 55.8 years (range $45-82$ years). These patients were seen between November 1999 and May 2003 in Hippokrateion General Hospital, Thessaloniki, Greece. The patients were divided in two groups. The main group included 76 perimenopausal patients with AUB who were taking sequential or continuous HRT or tibolone to relieve climacteric symptoms and to prevent osteoporosis. The mean age of these patients was 51.4 years (range $45-74$ years). The control group included 69 patients that presented with AUB during the postmenopausal period but were not taking any therapy and that were bleeding one year after the cessation of menses in women who did not receive HRT. The mean age of these patients was 57.6 years (range 48-82 years). The symptoms of the patients in the main group were menorrhagia in seven patients $(9.2 \%)$, irregular bleeding (metrorrhagia) in 38 patients $(50 \%)$, menorrhagia with irregular bleeding (menometrorrhagia) in 13 patients $(17.1 \%)$, dysmenorrhea in 12 patients $(15.7 \%)$, and postcoital bleeding in six patients $(7.8 \%)$.

The patients were asked to determine the phase of the sequential therapy when the bleeding episode had occurred, the duration and the severity of the episode, if there was a period of amenorrhea before the beginning of HRT, and if there were problems when receiving this kind of therapy. They did not take any other parallel therapies, and there were no risk factors for developing hyperplasia or cancer of the endometrium (like hypertension, diabetes or obesity).

Hormone replacement regimens included sequential oral or transdermal estrogen therapy with cyclical progestogens in 49 patients [ $2 \mathrm{mg}$ estradiol and $1 \mathrm{mg}$ norethisterone, Trisequens, Novo Nordisc (14 patients), 0.625 conjugated estrogens and $5 \mathrm{mg}$ medroxyprogesterone, Premelle cycle 5, Wyeth (11 patients), $50 \mathrm{mcg}$ estradiol and $0.25 \mathrm{mg}$ norethisterone, Estracomb TTS, Novartis (24 patients)], continuous oral estrogen with progestogens in 21 patients [ $2 \mathrm{mg}$ estradiol plus $1 \mathrm{mg}$ norethisterone, Kliogest, Novo Nordisc (nine patients), 0.625 conjugated estrogens plus $2.5 \mathrm{mg}$ medroxyprogesterone Premelle, Wyeth (12 patients)] and tibolone $2.5 \mathrm{mg}$ [Livial, Organon] in six patients. The mean duration of HRT usage was 1.8 years (range 6 months to 5 years). All patients had a bimanual gynaecological examination, a Pap smear when they had not had one in the last year, and a pelvic ultrasound before the diagnostic hysteroscopy was performed. Most hysteroscopies were performed with a 4-mm diameter rigid hysteroscope (Karl Storz, Germany) with $30^{\circ}$ fore-oblique lens and a 5.5-mm diagnostic sheath. A few hysteroscopies were performed with a $2.7-\mathrm{mm}$ diameter hysteroscope (ACMI Circon, USA) with a $3.5-\mathrm{mm}$ diagnostic sheath. Illumination was provided by a $300 \mathrm{~W}$ xenon cold light source through a fiber optic lead. The hysteroscopic images were viewed on a highresolution color monitor using a video camera. In all cases the uterine cavity was distended with one liter of normal saline wrapped in a pressure cuff (75$120 \mathrm{mmHg}$ ). Endometrial biopsies were taken with a very small metal curette or pipelle, and in a few cases with a hysteroscopic semi-flexible spoon or grasping forcep via a 7-mm operative sheath.

Patients were placed in the lithotomy position and the external genital organs, vagina and cervix were washed with antiseptic solution. A gynaecological examination was then performed; two gynaecological valves were placed in position, the anterior lip of the cervix was grasped with a tenaculum and the hysteroscope was attached to the external os. The valves were removed and the distention was initiated. The hysteroscope was guided inside the cervical canal under visual control via an atraumatic technique, using the distention of the normal saline and taking into account the $30^{\circ}$ fore-oblique view. The endometrial cavity and endometrium were inspected in detail starting from the tubal ostia, anterior, posterior and lateral uterine walls, using the rotation of the hysteroscope. Finally, the cervical os was inspected during the withdrawal of the hysteroscope. Each diagnostic hysteroscopy was followed by an endometrial biopsy. No anesthesia were used during the diagnostic hysteroscopies and endometrial biopsies, but if the patients felt discomfort or pain, the process was performed under sedation in the same operating room. Sedation was also used if the operative sheath was required for target biopsy, in minor operative procedures when the cervix was dilated up to $7-8 \mathrm{~mm}$, or when the resectoscope was used for the resection of a submucous myoma or a large endometrial polyp.

All of the hysteroscopic findings and the incidence of total uterine pathology for the two groups were compared using chi-square tests in order to assess statistical significance, with $P<0.05$ considered significant.

\section{Results}

Diagnostic hysteroscopy was performed successfully in $100 \%$ of patients under sedation when outpatient hysteroscopy was not possible, and intrauterine pathology was diagnosed in $52.6 \%$ of the cases in the main group. The most frequent and normal finding in this group was functional endometrium in 29 patients $(38.1 \%)$. Endometrial polyps were found in 19 patients $(25 \%)$, submucous fibroids in 11 patients $(14.5 \%)$, simple endometrial hyperplasia in nine patients $(11.8 \%)$ and complex hyperplasia or endometrial cancer in no patients. There was histologic confirmation of the hysteroscopic diagnosis in all cases, and outpatient hysteroscopy (no anesthesia) was used in $34.2 \%$ of the cases: 26 patients) while general anaesthesia (sedation) was used when there was need for cervical dilatation and during minor or major operative hysteroscopy $(65.8 \%)$. Endometrial biopsy with a small curette after diagnostic hysteroscopy with normal findings, or directed endometrial biopsy was performed in all cases of the study. In the control group the main hysteroscopic finding was atrophic endometrium in 28 patients $(40 . \%)$ and endometrial pathology in 30 patients $(43.4 \%)$. There were 
two cases of complex hyperplasia and one case of endometrial cancer in this group of patients. Outpatient hysteroscopy was performed in 21 patients $(30.4 \%)$. No serious complications (like uterine perforation or bleeding) occurred during the diagnostic hysteroscopies. We had three cases of vasovagal effects during outpatient hysteroscopy that were performed under sedation later. The hysteroscopic findings for the patients in this study are shown in Fig. 1.

Statistical significance between the hysteroscopic findings of the two groups was assessed by chi-square test with Yates' correction. Functional endometrium was found in 29 patients of the HRT group versus four patients of the control group $(P<0.001)$, endometrial polyps in 19 versus $10, P=0.17(\mathrm{NS})$, cervical polyps in three versus six, $P=0.2(\mathrm{NS})$, submucous myomas in 11 versus seven patients $P=0.6$ (NS), myomas and endometrial polyps in one versus two patients, $P=0.6$ (NS), atrophic endometrium in two of the HRT group versus 28 of the control group, $P<0.001$, endometritis or intrauterine adhesions in two versus three patients, $P=0.66$ (NS), simple hyperplasia without atypia in nine versus six patients, $P=0.7(\mathrm{NS})$, complex hyperplasia without atypia in two patients of the control group, $P=0.22$ (NS) and endometrial cancer was found in one patient of the control group, $P=0.47$ (NS). The incidence of detection of total intrauterine pathology between the two groups was $52.6 \%$ for the HRT group versus $43.4 \%$ for the control group. This difference is not significant $P>0.05$ (NS) after statistical analysis with the chi-square test $(P=0.27)$.

Histologic results confirmed the hysteroscopic diagnosis in all cases, as shown in Tables 1and 2. In each

Fig. 1 Hysteroscopic diagnosis for the patients of the two groups case of submucous myoma, the histologic result after endometrial biopsy with small curette was functional endometrium, but after resection it was established as a myoma. Also, in a few cases with atrophic endometrium there was not enough tissue for histology.

\section{Discussion}

Abnormal uterine bleeding during the perimenopausal and postmenopausal periods is common and a frequent cause for concern. There is a need for accurate diagnosis of endometrial pathology and a thorough evaluation of the uterine cavity in order to exclude endometrial neoplasia, a condition occurring with increasing incidence and possessing potentially serious consequences.

Our results show that diagnostic hysteroscopy and endometrial biopsy is a reliable procedure for assessing perimenopausal women taking HRT or women who present postmenopausal bleeding. There is an increased incidence of endometrial pathology diagnoses in women who present AUB while taking HRT. This incidence is also increased in women who present postmenopausal bleeding. The differences between the two groups in the detection of focal lesions or structural endometrial abnormalities are not statistically significant. In the perimenopausal women taking HRT (main group), functional endometrium is the main hysteroscopic finding and more common than endometrial polyps, hyperplasia or carcinoma. Also, in the postmenopausal women who did not take any hormone therapy (control group), atrophic endometrium is the main hysteroscopic finding, as would be expected. These differences between the two groups were statistically significant and the commonest diagnoses. This differentiation is important

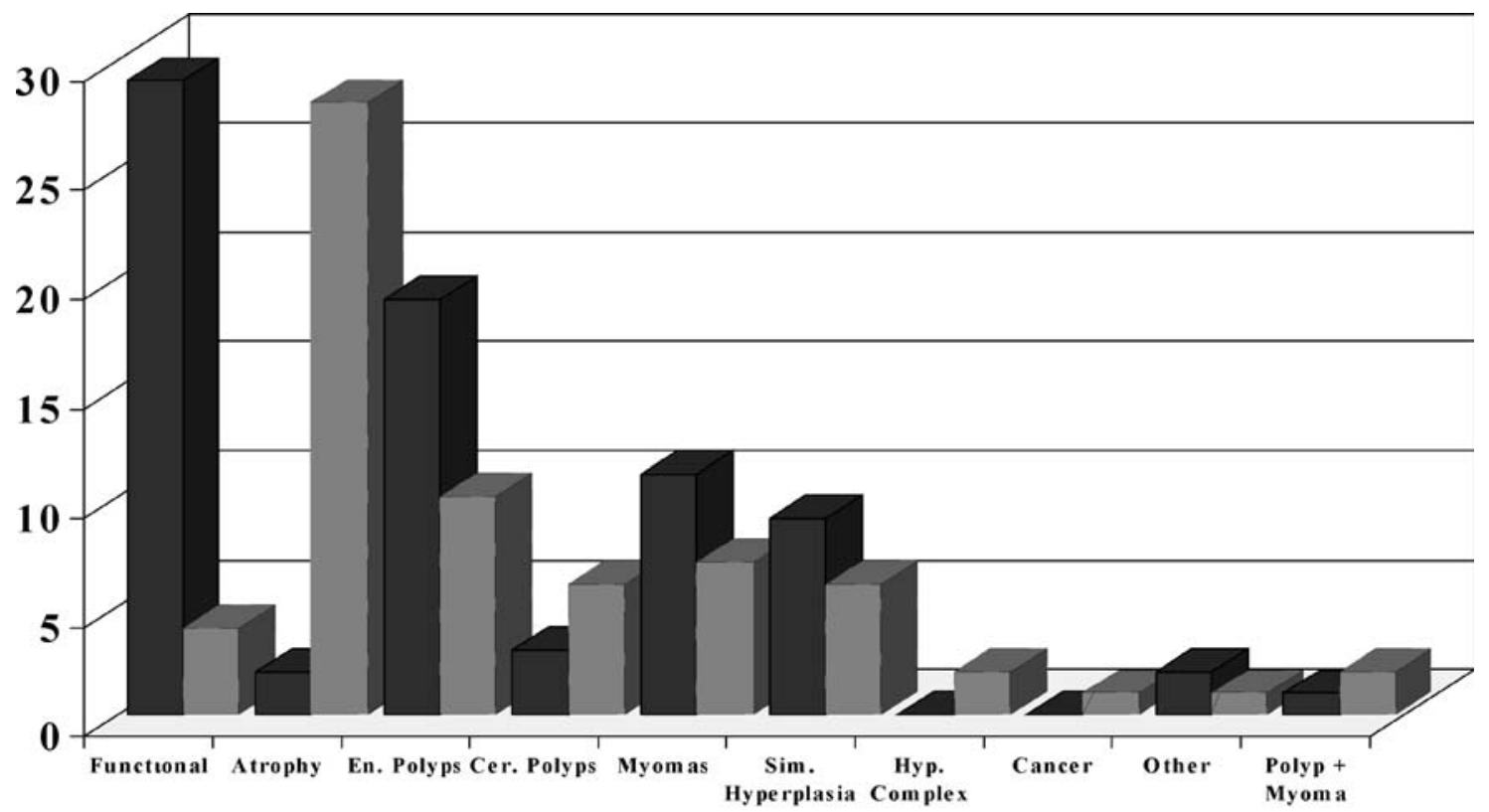

$\square$ Main group $\quad$ Contol group 
Table 1 Histologic findings for the main group (AUB on HRT)

\begin{tabular}{|c|c|c|c|c|c|c|c|c|c|}
\hline \multirow[t]{3}{*}{ Hysteroscopy } & \multirow[t]{3}{*}{ Patients } & \multicolumn{8}{|c|}{ Histologic diagnosis } \\
\hline & & \multicolumn{2}{|c|}{ Endometrium } & \multicolumn{2}{|l|}{ Polyp } & \multicolumn{2}{|c|}{ Hyperplasia } & \multirow[t]{2}{*}{ Cancer } & \multirow{2}{*}{$\begin{array}{l}\text { Insufficient } \\
\text { tissue/endometritis }\end{array}$} \\
\hline & & Functional & Atrophic & Cervical & Endometrial & Simple & Complex & & \\
\hline Normal & 29 & 29 & & & & & & & \\
\hline Atrophy & 2 & & 1 & & & & & & 1 \\
\hline Endometrial polyp & 19 & & & & 19 & & & & \\
\hline Cervical polyp & 3 & & & 3 & & & & & \\
\hline Submucous fibroid & 11 & 11 & & & & & & & \\
\hline Endometritis & 1 & & & & & & & & $1^{*}$ \\
\hline Hyperplasia & 9 & & & & & 9 & 0 & & \\
\hline Cancer & 0 & & & & & & & 0 & \\
\hline Intrauter. adhesions & 2 & & 2 & & & & & & \\
\hline
\end{tabular}

*Distinguishes the histologic diagnosis of endometritis from cases with insufficient tissue

Table 2 Histologic findings for the control group (postmenopausal bleeding)

\begin{tabular}{|c|c|c|c|c|c|c|c|c|c|}
\hline \multirow[t]{2}{*}{ Hysteroscopy } & \multirow[t]{2}{*}{ Patients } & \multicolumn{8}{|c|}{ Histologic diagnosis } \\
\hline & & Functional & Atrophic & Cervical & Endometrial & Simple & Complex & Cancer & $\begin{array}{l}\text { Insufficient } \\
\text { tissue/endometritis* }\end{array}$ \\
\hline Normal & 4 & 4 & & & & & & & \\
\hline Atrophy & 28 & & 25 & & & & & & 3 \\
\hline Endometrial polyp & 12 & & & & 12 & & & & \\
\hline Endometritis & 2 & & & & & & & & $2 *$ \\
\hline Hyperplasia & 8 & & & & & 6 & 2 & & \\
\hline Cancer & 1 & & & & & & & 1 & \\
\hline Intrauter. adhesions & 1 & & 1 & & & & & & \\
\hline
\end{tabular}

*Distinguishes the histologic diagnosis of endometritis from cases with insufficient tissue

because it changes the treatment options for the patients. The increasing number of postmenopausal women being treated with HRT may be associated with an increase in the number of women presenting with AUB. Identification of the exact endometrial pathology by hysteroscopy and endometrial biopsy and the subsequent treatment of patients may avoid the need for discontinuation of HRT, especially in the first few months of the therapy when most episodes of AUB usually occur [6-8]. The main type of AUB that occurred in women receiving sequential HRT was relatively heavy bleeding after the progestogen phase or a shorter breakthrough bleeding for a few days earlier in the cycle. In women receiving continous HRT, the bleeding episodes were shorter in duration of the type of spotting at any time. A high incidence of endometrial pathology observed in the patients of the main and control study groups $(52.6 \%$ and $43.4 \%$, respectively) has also been reported by others $[9,10]$ too. The reason for this may be that AUB in perimenopausal and postmenopausal years is managed traditionally by dilatation and curettage, a method with lower sensitivity in the detection of focal endometrial lesions compared to hysteroscopy [11, 12]. Outpatient hysteroscopy in our study was performed in over
$30 \%$ of the patients, but this percentage is increasing gradually with newer, narrower, instruments and staff training in diagnostic and operative hysteroscopy [13, 14]. Hysteroscopy can be performed on an outpatient basis or as a day case procedure with high acceptability and satisfaction from patients $[15,16]$. It also has the advantage of being able to treat endometrial pathology at the same time as diagnosing endometrial polyps or submucous fibroids, and endometrial ablation is an alternative to hysterectomy in many cases [17-20]. The development of a one-stop perimenopausal bleeding clinic in our department in the near future will offer patients evaluation of the uterine cavity without hospitalization. It will be essential for the treatment of all patients suffering from perimenopausal or postmenopausal bleeding, and its success is already known from other studies [21-24].

In conclusion, the management of perimenopausal and postmenopausal bleeding may be based on hysteroscopy in combination with endometrial biopsy, because it is a reliable tool for precisely evaluating the topography of the uterine cavity and the nature of the endometrium. An increased incidence of intrauterine pathology is observed in women receiving HRT with 
AUB and in those who present postmenopausal bleeding.

\section{References}

1. McKinlay SM, Brambilla DJ, Posner JG (1992) The normal menopause transition. Maturitas 14:103

2. Treolar AE, Boynton RE, Borghild GB, Brown BW (1967) Variation of the human menstrual cycle through reproductive life. Int J Fertil 12:77

3. Richardson SJ, Senikas V, Nelson JF (1987) Follicular depletion during the menopausal transition-evidence for accelerated loss and ultimate exhaustion. J Clin Endocrinol Metab 65:1231

4. Gougeon A, Ecochard R, Thalabard JC (1994) Age-related changes of the population of human ovarian follicles: increase in the disappearance rate of non-growing and early-growing follicles in aging women. Biol Reprod 50:653

5. Abu JI, Habiba MA, Baker R, Halligan AW, Naftalin, NJ, Hsu R, Taub N (2001) Quantitative and qualitative assessment of women's experience of a one-stop menstrual clinic in comparison with traditional gynaecology clinics. BJOG 108(9):9939

6. Hahn RG, Nachtigal RD, Davies TC (1984) Compliance difficulties with progestin supplemented replacement therapy. J Fam Pract 18:411-414

7. Nachtigall LE (1990) Enhancing patient compliance with hormone replacement therapy at menopause. Obstet Gynecol 75(Supp):77-80

8. Al-Azzawi F, Habiba M (1994) Regular bleeding on hormone replacement therapy: a myth? Br J Obstet Gynaecol 101:661662

9. Nagele F, O'Connor H, Basket TF, Davies A, Magos AL (1996) Hysteroscopy in women with abnormal uterine bleeding on hormone replacement therapy: a comparison with postmenopausal bleeding. Fertil Steril 65:1145-1150

10. Downes E, Al-Azzawi F (1993) The predictive value of outpatient hysteroscopy in a menopause clinic. $\mathrm{Br} \mathrm{J}$ Obstet Gynaecol 100:1148-1149

11. Goldrath MH, Sherman AI (1985) Office hysteroscopy and suction curettage: can we eliminate the hospital diagnostic dilatation and curettage? Am J Obstet Gynecol 152:220-229
12. Emanuel MH, Wamsteker K, Lammes FB (1997) Is dilatation and curettage obsolete for diagnosing intrauterine disorders in premenopausal patients with persistent abnormal uterine bleeding? Acta Obstet Gynecol Scand 76(1):65-68

13. Campo R, Van Belle Y, Rombauts L, Brosens I, Gordts S (1999) Office mini-hysteroscopy. Hum Reprod Update 5(1):7381

14. Nagele F, O'Oconnor H, Davies A, Badawy A, Magos A (1996) 2500 outpatient diagnostic hysteroscopies. Obstet Gynecol 8(1):87-92

15. Kremer K, Duffy S, Moroney M (2000) Patient satisfaction with outpatient hysteroscopy versus day case hysteroscopy: randomised controlled trial. BMJ 320:279-282

16. Abu JI, Habiba MA, Baker R, Halligan AW, Naftalin NJ, Hsu R, Taub N (2001) Quantitative and qualitative assessment of women's experience of a one-stop menstrual clinic in comparison with traditional gynaecology clinics. BJOG 108(9):993-999

17. Loverro G, Bettocchi S, Cormio G, Nicolardi V, Greco P, Selvaggi L (1999) Transvaginal sonography and hysteroscopy in postmenopausal uterine bleeding. Maturitas 33(2):139-144

18. Magos AL, Bowmann R, Lockwood GM, Turnbull AC (1991) Experience with the first 250 endometrial resections for menorrhagia. Lancet 337:1074-1078

19. Al-Azzawi F (1999) Prediction of successful outcome following hysteroscopic laser myomectomy in women with heavy and unscheduled bleeding. Climacteric 2(Suppl):289

20. Cravello L, de Montgolfier R, D'Ercole C, Boubli L, Blanc B (1996) Hysteroscopic surgery in postmenopausal women. Acta Obstet Gynecol Scand 75:563-566

21. Corson SL (1995) Hysteroscopic diagnosis and operative therapy of submucous myoma. Obstet Gynecol Clin North Am 22:739-755

22. Zupi E, Luciano AA, Valli E, Marconi D, Maneschi F, Romanini C (1995) The use of topical anesthesia in diagnostic hysteroscopy and endometrial biopsy. Fertil Steril 63:414-416

23. Pahal G, Hart R, Magos A (1998) Gold standard of care for menstrual problems: the one-stop clinic. Br J Obstet Gynaecol 105(Suppl 17):4

24. Baskett TF, O'Connor H, Magos AL (1996) A comprehensive one stop menstrual problem clinic for diagnosis and management of abnormal uterine bleeding. Br J Obstet Gynaecol 103:76-77 